\title{
Priority effects determine how dispersal affects biodiversity in seasonal metacommunities
}

\author{
Heng-Xing Zou ${ }^{1 *}$, Volker H. W. Rudolf ${ }^{2}$
}

Program in Ecology and Evolutionary Biology, Department of BioSciences, Rice University,

Houston, TX 77005

*Corresponding author. Emails: ${ }^{\text {[hengxingzou@ @ice.edu, }}{ }^{2}$ volker.rudolf@ rice.edu.

Statement of authorship: H.-X.Z. conceived the idea, designed, and performed the research with assistance from V.H.W.R. H.-X.Z. wrote the first draft, and both authors contributed to revisions.

Data accessibility: No new data were used. Code is available at GitHub

(https://github.com/hengxingzou/Zou2022bioRXiv) during the peer review process and will be archived to an online repository upon acceptance.

Running title: Priority effects in Metacommunities

Keywords: priority effects, metacommunity, dispersal-diversity relationships, phenology, seasonal variation

Number of words in the abstract: 150

Number of words in the main text: 4956

Number of references: 55

Number of figures: 6 


\section{Abstract}

2 The arrival order of species frequently determines the outcome of their interactions. This

3 phenomenon, called the priority effect, is ubiquitous in nature and determines local community

4 structure, but we know surprisingly little about how it influences biodiversity across different

5 spatial scales. Here, we use a seasonal metacommunity model to show that biodiversity

6 patterns and the homogenizing effect of high dispersal depend on the specific mechanisms

7 underlying priority effects. When priority effects are driven by a numeric advantage, diversity

8 measurements are highly sensitive to initial conditions and high dispersal homogenizes

9 communities. However, when local priority effects arise from phenological shifts that alter

10 species interaction strength (trait-mediated priority effects), local, regional, and temporal

11 diversity are higher and are surprisingly robust to variation in dispersal and initial conditions,

12 given spatiotemporal variations in phenology. Our results highlight that accounting for the

13 mechanisms underlying priority effects is fundamental to understanding the maintenance of

14 regional biodiversity. 


\section{Introduction}

Dispersal promotes the exchange of individuals between communities and thus links local population and community dynamics to regional patterns of species distributions. As a consequence, dispersal can play a key role in shaping biodiversity patterns at both local and regional scales (Kerr et al. 2002; Cadotte 2006; Grainger and Gilbert 2016). However, the relationship between dispersal and biodiversity patterns is also shaped by local conditions and processes, including biotic interactions (e.g. Kneitel and Miller 2003; Shurin et al. 2004;

Altermatt et al. 2011; Carrara et al. 2012; Vanschoenwinkel et al. 2013; Catano et al. 2017).

Although recent studies identify local competitive interactions as a major contributor to dispersal-diversity relationships (Pu and Jiang 2015; Lu 2021; Miller and Allesina 2021), one ubiquitous biotic process in metacommunities, the temporal sequence of community assembly, has been largely neglected.

Communities are not assembled all at once; instead, species arrive at different times and differences in the sequence of arrival times can alter the outcome of species interactions and thereby also community composition. Such priority effects (or historical contingencies) are widespread in nature and occur in systems ranging from microbes through plants and vertebrates (Alford and Wilbur 1985; Drake 1991; Kardol et al. 2013; Fukami 2015). In a metacommunity context, priority effects become important because they can alter the fate of immigrating individuals into a patch. For instance, with early arriver advantage, individuals that arrive at a patch may either successfully establish themselves if they arrive first, or they could perform poorly and even be excluded by earlier arriving competitors. By altering the outcome of local competitive interactions, priority effects have the potential to shape the relationship between dispersal and diversity patterns at local and regional scales. Although 
38 several theories predict that priority effects could affect how regional dispersal shapes

39 biodiversity (e.g. Shurin et al. 2004; Fukami 2015; Grainger and Gilbert 2016; Miller and

40 Allesina 2021), few have incorporated the diverse mechanisms that can generate priority

41 effects in natural systems. Yet accounting for these mechanisms may be key to explaining the

42 often-unexpected dispersal-diversity relationships found in different systems.

43 Priority effects can be generated by at least two fundamentally different mechanisms,

44 broadly categorized as "numeric" or "trait-mediated" (Rudolf 2019; Zou and Rudolf 2020).

45 Numeric priority effects arise from the positive frequency dependence of the species with

46 higher abundance (Ke and Letten 2018). As one species arrives early, it is more likely to

47 establish a numeric advantage over late arrivers and maintain this advantage through a positive

48 feedback. On the other hand, trait-mediated priority effects assume that per-capita interactions

49 between species change with their relative arrival times (Rudolf 2019). In nature, this occurs

50 through altered traits that could affect species interactions, such as size and behavior, or

51 modified environment from the difference in arrival times (Poulos and McCormick 2014;

52 Rasmussen et al. 2014; Rudolf 2018; Blackford et al. 2020). These changes in interacting traits

53 often result in a change of species' competitive interactions that correlate with their relative

54 arrival times (Rudolf 2019; Zou and Rudolf 2020). Therefore, if trait-mediated priority effects

55 occur, the competitive outcome of early and late arrivers are not only determined by their

56 numeric difference but also their relative arrival times.

57 Differences in local community dynamics between different types of priority effects also

58 have the potential to alter local and regional dispersal-diversity relationships. With numeric

59 priority effects, positive frequency dependence could enable the more abundant early arriver to

60 spread over the landscape through dispersal and competitively exclude late arrivers. High 
61 dispersal will therefore decrease both local and regional diversity because one or a few

62 regionally dominant species will eventually establish and maintain a numeric advantage in all patches, similar to the classic prediction that dispersal homogenizes (Tilman 1994; Mouquet and Loreau 2003; Leibold et al. 2004; Vanoverbeke et al. 2016; Fodelianakis et al. 2019). Following this premise, alpha diversity is likely highest at intermediate dispersal while beta and gamma diversity likely decreases with dispersal (Mouquet and Loreau 2002; 2003; Cadotte 2006). However, regional diversity may be retained even under high dispersal if priority effects are driven by trait-mediated mechanisms, such as when early arrivers alter the environment or rapidly adapts to the habitat (Shurin et al. 2004; Fukami 2015; Grainger and Gilbert 2016; Leibold et al. 2019; Miller and Allesina 2021). In these cases, competitive outcomes are not only driven by relative abundances but also relative arrival times. Early arrivers therefore will not be excluded even if other species are more abundant. This effect of relative arrival times should be particularly important in systems with periodic species assembly ("seasonal" communities), as the frequent assembly of local communities creates many opportunities for spatial and temporal variations in arrival orders (Sheriff et al. 2011; Diez et al. 2012; Theobald et al. 2017; Carter et al. 2018; Rudolf 2019). In these seasonal systems, trait-mediated priority effects and natural variations in species phenology could lead to high temporal turnover of local and regional community composition despite the homogenizing effect of dispersal. Thus, local priority effects and variations in phenology should strongly shape the dispersal-diversity relationship in seasonal metacommunities, but the specific relationship should depend on the mechanism of priority effects.

Here, we use a spatially implicit, multispecies metacommunity model to reveal how different mechanisms of priority effects interact with dispersal to shape biodiversity at local 
84 and regional scales in competitive metacommunities. We modeled the system with realistic

85 spatial and temporal variations in phenology and compared the observed dispersal-diversity

86 relationships with numeric and trait-mediated priority effects across different initial

87 distributions of population. We asked the following questions: (1) How will different

88 mechanisms of priority effects change dispersal-diversity relationships? (2) When will local

89 and regional diversity be maintained under high dispersal? (3) How sensitive are patterns of

90 biodiversity to initial heterogeneity in population sizes among local communities?

\section{Methods}

Model Setup

We modeled natural habitats with seasonal disturbance, such as annual grassland or ephemeral ponds, in which species survive periodic, unfavorable environments (e.g., winter, droughts) and reemerge at the beginning of the next season. We define a season as the growth period between a previous disturbance to the next disturbance. For instance, a "season" for annual plants would start with the emergence in spring or summer and end with senescence in fall or winter, while a "season" for ephemeral ponds would be when they hold water. At the beginning of the season, each species emerges from dormancy in a patch at an assigned time.

101 Patches are homogeneous throughout the landscape and are not spatially structured. The average time of emergence in a season ( $\bar{p}$, for phenology) is fixed for each species, but the

103 exact emergence time is randomly determined at each patch by drawing from a uniform

104 distribution centered around the species-specific average time of emergence (Figure 1).

105 Therefore, despite fixed average times of emergence, the exact order of arrival in the model 106 varies among patches and between seasons. This reflects variations in the phenology of co- 
107

108

109 Carter et al. 2018).

110

111

112

113

114

115

116

117

118

119

120

121

122

123

124

125

126

127

128

occurring species arising from species-specific responses to environmental stochasticity across space and time in natural systems (Sheriff et al. 2011; Diez et al. 2012; Theobald et al. 2017;

Within each season, local population dynamics in a given patch are determined with a modified Beverton-Holt model (Rudolf 2019):

$$
N_{i}(t+1)=\frac{\lambda_{i} N_{i}(t)}{1+\sum_{j=1}^{n} \alpha_{i j} \lambda_{i} N_{j}(t)}+s_{i} N_{i}(t)(\text { Eqn. 1) }
$$

where the population of the next season, $N_{i}(t+1)$, is determined by population growth of species $i, \lambda_{i} N_{i}(t)$, which negatively scales with densities of all species with respective competition coefficients $\alpha_{i j}$, and the proportion surviving to the next season, $s_{i} N_{i}(t)$. All interspecific competitions are pairwise.

If the community is driven by trait-mediated priority effects, interspecific competition is a function of the relative time of emergence:

$$
\alpha_{i j}(\Delta p)=\frac{B}{1+e^{(d-\Delta p) / c}}(\text { Eqn. 2) }
$$

where $\Delta p$ is the relative time of emergence between species $i$ and $j$, calculated as $t_{j}-t_{i}$, and $B, d$ and $c$ are constants that determine the asymptotic maximum, midpoint (when two species emerge simultaneously), and shape of the sigmoidal function. This nonlinear competitionphenology function assumes an early arriver advantage and has been validated by several empirical studies in animal and plant systems (Shorrocks and Bingley 1994; Rudolf 2018; Blackford et al. 2020). If the community is only driven by numeric priority effects, the interspecific competition does not depend on the relative time of emergence, and $\Delta p$ is effectively 0 . In this scenario, the interspecific competition becomes a constant: $\alpha_{i j}(0)=\frac{B}{2}$. We modeled the dispersal process as follows: first, $D$ emigrating patches are randomly 
129 selected, and emigrating populations of each species are determined by the number of

130 successes of a binomial draw with $n$ trials and probability $r ; n$ is the population of that species

131 in that patch (Thompson et al. 2020). Emigrants are then pooled by species and redistributed to

$132 D$ randomly selected patches; $D$ is hereafter termed patches with dispersal. This spatially

133 implicit method has been widely used in empirical studies that often manually transfer

134 individuals between patches (Grainger and Gilbert 2016) and theoretical models (e.g.

135 DeMalach et al. 2021) and assumes that dispersal is not affected by distance or patch choice.

136 After dispersal, the season ends with a disturbance event, in which an end-of-season survival

137 rate $\left(s_{i}\right.$ in Eqn. 1) determines the proportion surviving to the next season.

\section{Model Simulations}

Although our model can be extended to any number of species and patches, we simulated

141 metacommunities with 5 competing species and 50 patches. This number of species allows for

143 Each simulation was run for 100 seasons, long enough for us to discern population dynamics

144 driven by competition, as patterns such as extinction and dominance qualitatively stabilized

145 within 25-50 seasons, and we confirmed these observed patterns by simulating each set of

146 parameters 50 times. In our model, dispersal is dependent on both dispersal rate $(r)$ and patches

147 with dispersal $(D)$. Therefore, we varied them independently across a wide range (Table S1) to

148 ensure that we observe the full range of possible dynamics under biologically realistic

149 scenarios. We considered these two parameters as separate processes since they reflect different

150 aspects of dispersal. The proportion of population being dispersed under the lowest

151 combination of dispersal parameters $(r=0.001, D=10)$ is approximately $1 \%-2 \%$, while this 
152 proportion is $20-30 \%$ under the highest combination $(r=0.1, D=40)$.

153 We created a competitive hierarchy across all five species by differentiating intraspecific

154 competition (intensity of self-limitation), with species 1 having the highest and species 5

155 having the lowest intraspecific competition coefficient. We set the average order of emergence

156 time in reverse order to the competitive hierarchy, where species 1 emerges the earliest and

157 species 5 the latest. Additional simulations show whether the competitive hierarchy matches

158 with the order of emergence does not qualitatively change results. Note that with trait-mediated

159 priority effects interspecific competition (and thus competitive hierarchies) also depend on the

160 order of emergence (Eq. 2). We chose intraspecific competition coefficients that are smaller

161 than baseline interspecific competition coefficients $\left(\frac{B}{2}\right)$ to promote numeric priority effects, but

162 our results are not sensitive to higher intraspecific competition strengths, which promote

163 coexistence as shown by additional simulations. We assumed that each season consists of one

164 generation, dispersal happens once at the end of a season, all species have equal dispersal rates

$165(r)$, and no patch undergoes extinction. To determine the robustness of observed patterns, we

166 relaxed each of the assumptions and did not find a qualitative change in observed differences in

167 population dynamics and dispersal-diversity relationships between mechanisms of priority

168 effects. Specific model parameters are listed in Table S1.

169 All simulations were performed in R version 4.1.1 (R Core Team 2021). Simulation code

170 is available at GitHub (https://github.com/hengxingzou/Zou2022bioRXiv) during the peer

171 review process and will be archived to an online repository upon acceptance.

\section{Measuring Biodiversity}

174 We examined dispersal-diversity relationships by calculating local species richness (\# of 
175 species in each patch; alpha diversity), dissimilarity in community composition among patches

176 (beta diversity), and regional species richness (\# of species in the metacommunity; gamma

177 diversity) at the end of simulations. Alpha diversity was averaged among all patches within

178 each repetition. We quantified beta diversity (dissimilarity in community composition based on

179 numeric abundances of species) by averaging all pairwise Bray-Curtis distances between

180 patches using the R package vegan (Oksanen et al. 2013).

181 We also calculated temporal beta-diversity to quantify changes in metacommunity

182 composition over time using the R package adespatial (Dray et al. 2018). Specifically, we

183 calculated the dissimilarity ( $D$ metric in Legendre 2019) in species composition of a

184 metacommunity between consecutive time steps separated by 10 seasons. A high dissimilarity

185 index indicates that relative abundances of species vary greatly over time.

187 Scenarios and Results

The heterogeneity in initial population densities among patches strongly affects measured

189 dispersal-diversity patterns (Grainger and Gilbert 2016; Lu 2021) and priority effects are likely

190 to further amplify these differences. Therefore, we set three scenarios that varied in initial

191 relative abundances of species within and across patches and compared the resulting

192 biodiversity measures with and without trait-mediated priority effects.

\section{Scenario 1: Initial populations are separated across patches}

195 We first consider a simple baseline scenario in which each patch is initially colonized by

196 only one species and each species colonized the same number of patches. Therefore, the initial

197 alpha diversity is low (one species per patch), while beta and gamma diversities are high. 
198 Results of this scenario immediately highlight the difference between dynamics with and

199 without trait-mediated priority effects. With numeric priority effects, the initial resident

200 remains dominant in a given patch for low and medium levels of dispersal since the number of

201 immigrants is small compared to the resident's population (Figure 2A, 2C). This pattern only

202 changes when dispersal (dispersal rate, $r$, and patches with dispersal, $D$ ) is high enough to boost

203 immigrant populations to a size that allows them to overcome the numeric advantage of the

204 resident species (Figure 2E). The difference in final population size between species simply

205 reflects species-specific differences in strength of intraspecific competition (Figure 3A, 3C),

206 except when high dispersal homogenizes the metacommunity (Figure 3E). In contrast, with

207 trait-mediated priority effects, immigrants can successfully invade and even achieve dominance

208 in new patches (Figure 2B, 2D). This invasion is possible because the relative timing of

209 emergence (and not initial densities) determines per-capita effects and thus competitive

210 dominance. Therefore, metacommunities with trait-mediated priority effects show a clear

211 hierarchy of final population and regional dominance, reflecting early-arriver advantage

212 (Figure 3B, 3D, 3F).

213 These differences in patch dynamics between both types of priority effects (numeric vs.

214 trait-mediated) strongly affect the local and regional dispersal-diversity relationships. Alpha

215 diversity shows a clear hump-shaped relationship with total dispersal (combined effect of $r$ and

$216 D$ ) in metacommunities with numeric priority effects (Figure 4A). At a lower dispersal rate $(r=$

217 0.001) alpha diversity increases with higher $D$ (more patches with dispersal) but decreases with

218 higher $D$ at the highest dispersal rate $(r=0.1$; Figure S6-8A). In contrast, alpha diversity

219 monotonically increases with dispersal (both $r$ and $D$ ) in metacommunities with trait-mediated

220 priority effects (Figure 4B; Figure S6-8A). 
With numeric-mediated priority effects, beta diversity decreases with higher dispersal

222 (both $D$ and $r$ ), indicating that dispersal homogenizes the metacommunity and leads to regional

223 dominance of a single species (Figure 5A; Figure S6-8D). This negative dispersal-diversity

224 relationship is present but weaker in systems with trait-mediated priority effects (Figure 5B).

225 Furthermore, the overall dissimilarity is lower with trait-mediated compared to numeric

226 priority effects, as patches quickly gain species from the initial monoculture (Figure 5A-B;

227 Figure S6-8D). Gamma diversity remains high in metacommunities with numeric priority

228 effects, as some species are locally extinct, but no species goes extinct regionally unless overall

229 dispersal is very high $(r=0.1, D=40$, or $80 \%$ of all patches; Figure S5A, S6-8G). With trait-

230 mediated priority effects, no regional extinction occurs, and gamma diversity is always at

231 maximum even under high overall dispersal (Figure S5A, Figure S6-8G).

\section{Scenario 2: Initial populations are equal across all patches}

234 We next explored a scenario where all five species start with equal population sizes in all

235 patches. This starting condition is often used in lab experiments (Grainger and Gilbert 2016).

236 Similar to Scenario 1, we found a striking difference in population dynamics with and without

237 trait-mediated priority effects. With numeric priority effects, the competitively superior species

238 (species 5) dominates most patches, and the dominance is promoted by dispersal, although in

239 rare cases other species can rise to dominance as well (Figure S1C). With trait-mediated

240 priority effects, the observed pattern is very similar to that of Scenario 1: all species coexist,

241 the dominant species in a patch changes quickly over time, but the species with the earliest

242 time of emergence on average (species 1) still has the largest total population (Figure S1, S3).

243 In this scenario, we also found a weak hump-shaped pattern between alpha (Figure 4C; 
244 Figure S6-8B), beta (Figure 5C; Figure S6-8E), and gamma (Figure S5, S6-8H) diversity and

245 overall dispersal in metacommunities with numeric priority effects. However, alpha diversity of

246 metacommunities with trait-mediated priority effects consistently increases with higher

247 dispersal (Figure 4D; Figure S6-8B). This results in a concurrent decrease in beta diversity

248 (Figure 5D; Figure S6-8E), although the change of both diversity values is small compared to

249 that with numeric priority effects. Finally, gamma diversity remains constant since no regional

250 extinction occurs with trait-mediated priority effects (Figure S5C; Figure S6-8H).

Scenario 3: Initial populations vary randomly across patches

Finally, we consider a scenario where populations of each species vary among patches,

which is often the case in field experiments with heterogeneous starting conditions (Kneitel

conditions by allowing all five species to occupy all patches, but their initial population is

257 randomly drawn from a normal distribution with a mean of 50 (initial population used in

258 Scenario 1 and 2) and a standard deviation of 10.

259 In metacommunities with numeric priority effects, the species with an initial numeric

260 advantage may be able to maintain its dominance in a given patch, but increasing dispersal

261 (high $D$ or $r$ ) also allows competitively stronger species to replace the initially most abundant

262 species over time (Figure 2E). When $r$ or $D$ is small, the final total population of each species

263 reflects their initial population and strength of intraspecific competition (Figure S4A, C), but

264 higher dispersal quickly homogenizes the metacommunity (Figure S4E). In contrast,

265 metacommunities with trait-mediated priority effects again display very similar patterns to

266 those in Scenarios 1 and 2, with highly variable dynamics over time regardless of spatial 
267 dispersal (Figure S2) and species 1 (earliest emergence) having the highest local dominance

268 (Figure S4).

269 With numeric priority effects, alpha diversity displays a unimodal relationship with

270 dispersal (Figure 4E), while it consistently increases with dispersal with trait-mediated priority

271 effects (Figure 4F). Higher dispersal contributes to more regional extinction and thus reduced

272 gamma diversity with numeric priority effects (Figure S5E). In contrast, gamma diversity

273 remains at maximum with trait-mediated priority effects, even under high dispersal levels

274 (Figure S5F). Metacommunities with both numeric and trait-mediated priority effects show

275 decreasing beta diversity with higher dispersal, although the change is much more obvious for

276 those with numeric priority effects, indicating that they have received a stronger homogenizing

277 effect from dispersal (Figure 5E-F; Figure S6-8F).

\section{Temporal Turnover of Metacommunity}

The difference between local population dynamics of numeric and trait-mediated priority

281 effects is further reflected by the temporal turnover of metacommunities. The initial decrease of

282 dissimilarity index in all cases is due to the stabilization process of local communities (see

283 Figure 2). In metacommunities with numeric priority effects, the temporal trend of beta

284 diversity depends on the dispersal rate $r$ and initial scenarios (Figure 6; Figure S9-10). Under

285 Scenario 1, low dispersal results in little (but nonzero) temporal turnover (Figure 6A) after the

286 initial drop. This indicates the persistent but extremely low turnover brought by dispersal,

287 while intermediate and high dispersal results in higher turnover due to a higher population flow

288 in and out the patch (Figure 6C, 6E). Note that even when the metacommunity is dominated by

289 a single species (as in Figure 2E), its population still fluctuates frequently due to high dispersal 
rates, leading to relatively high temporal turnover (Figure 6E). However, with trait-mediated

291 priority effects, metacommunities show a consistently high temporal beta diversity under low,

292 medium, and high dispersal rates and across all initial scenarios (Figure 6; Figure S9-10).

293 Under Scenario 1, even with the lowest dispersal rate, the temporal turnover is still higher than

294 with numeric priority effects only (Figure 6A, 6B). This observation corresponds to the high

295 species turnover observed in population dynamics regardless of initial conditions. Overall,

296 these temporal trends are consistent with the observed local population dynamics and highlight

297 the importance of the specific mechanism that produces priority effects for biodiversity

298 patterns across all spatial and temporal scales.

\section{Discussion}

Priority effects are ubiquitous in natural systems, yet their role in shaping biodiversity

302 patterns is still poorly understood (Fukami 2015). Here we show that priority effects can play a

303 key role in shaping local and regional biodiversity of seasonal communities and how they are

304 influenced by dispersal. However, the shape of this dispersal-diversity relationship depends on

305 the mechanisms underlying the priority effects, i.e., whether they are generated by numeric or

306 trait-mediated effects. When relative arrival times (phenological differences) vary across space

307 and time, metacommunities with numeric priority effects are highly sensitive to the initial

308 conditions and become homogenized at high levels of dispersal with low local and regional

309 diversity. In contrast, trait-mediated priority effects promote local and regional species

310 coexistence even under very high levels of dispersal and the patterns are highly robust to

311 variation in initial conditions. Together, these results provide novel insights into how priority

312 effects shape the dispersal-diversity relationships in nature and highlight the importance of 
313 considering the seasonal nature of local community assembly to predict regional patterns.

\section{Positioning Priority Effects in Current Dispersal-Diversity Framework}

316 Classic models predict that the dispersal-diversity relationship should be maximized at

317 intermediate dispersal, while between-patch and regional diversity should constantly decrease

318 with dispersal (Mouquet and Loreau 2002; 2003). This prediction still qualitatively holds when

319 local dynamics are driven by numeric priority effects in our model, but with different

320 mechanisms. In classical models, diversity peaks at intermediate dispersal because species are

321 present in both source and sink habitats, and the population in the latter can be rescued by

322 immigration from the former (Leibold et al. 2004). Traditionally, source-sink dynamics are

323 expected to be driven by spatial heterogeneity in habitat conditions (Pulliam 1988). However,

324 source-sink dynamics are still possible with numeric priority effects, even in the absence of any

325 spatial heterogeneity in habitat quality because the numeric advantage of resident species poses

326 a barrier for rare immigrating species to establish themselves. These patches serve as sources

327 for numerically dominant species and sinks for all other (immigrating) species. At low to

328 moderate dispersal rates, numeric priority effects thus maintain high beta diversity within

329 metacommunities. This is especially visible under Scenarios 1 and 3, where each species is

330 initially numerically dominant in some patches.

331 This source-sink dynamic diminishes when dispersal is high enough for competitively

332 superior species to reach patches in large enough numbers to overcome the numeric priority

333 effects of residents and eventually exclude them both locally and regionally. Once this

334 threshold is reached, dispersal works as a homogenizing force that decreases local and regional

335 diversity (Mouquet and Loreau 2003; Grainger and Gilbert 2016; Catano et al. 2017). 
336 Furthermore, we also found that the shape of this relationship is highly sensitive to initial

337 conditions (i.e., how species are distributed among patches) in metacommunities with numeric

338 priority effects. This result is not previously emphasized in theory (but see Lu 2021) and could

339 at least partly help explain why the diversity-stability relationship differs among studies that

340 use different initial settings (Grainger and Gilbert 2016).

341 On the contrary, diversity patterns in metacommunities with trait-mediated priority effects

342 are much less affected by dispersal or initial conditions. In these metacommunities, immigrants

343 may successfully colonize a patch regardless of their numeric disadvantage as their

344 colonization success is more dependent on the order of arrival (emergence). If a species arrives

345 early enough, its early arrival advantage (relative increase in interspecific competitive ability)

346 can overcome its numeric disadvantage and allow it to invade the resident species. Given the

347 temporal and spatial variation in arrival times (species phenology), trait-mediated priority

348 effects can therefore promote local and regional coexistence of species by preventing

349 competitive exclusion and homogenization at a high dispersal rate. This result agrees with a

350 recent study that models patch memory, which is similar to trait-mediated priority effects via

351 habitat modification (the previous resident modifies the habitat and therefore affects

352 colonization success of the late arriver): with priority effects, any number of species stably

353 coexist despite dispersal (Miller and Allesina 2021).

354 The trait-mediated priority effect in our model appears conceptually similar to processes

355 implemented by some previous models (e.g. biotic heterogeneity in Shurin et al. 2004;

356 monopolization effects by local adaptation in Vanoverbeke et al. 2016). Yet, they found that

357 positive feedback of early arrivers enables their regional dominance. This result represents

358 what we found in metacommunities with high dispersal and numeric, but not trait-mediated 
359 priority effects. This discrepancy arises because we incorporated seasonality and realistic

360 spatial and temporal variations of arrival order in our model. These variations lead to a constant

361 reshuffling of competitive hierarchies across time and space that reduces or prevents local and

362 regional extinction, as predicted by previous theoretical results in non-spatial models (Rudolf

363 2019; Zou and Rudolf 2020). When aggregated on a spatial or temporal scale, this reshuffling

364 of competitive hierarchies can also lead to intransitive competition, which is predicted to

365 stabilize multispecies systems (Allesina and Levine 2011; Levine et al. 2017).

366 Our results provide a theoretical basis for interpreting many empirical results, especially

367 those explicitly considering the role of priority effects. For instance, experiments in freshwater

368 protists found that community composition in homogeneous patches was strongly correlated to

369 the temporal sequence of assembly despite dispersal (Pu and Jiang 2015). Our results suggest

370 that this counterintuitive pattern can be easily explained by trait-mediated priority. Consistent

371 with this prediction, Pu and Jiang (2015) reported changes in interspecific competition based

372 on arrival orders of species, indicating the presence of trait-mediated priority effects. Our

373 observations also corroborate the result that local priority effects in nectar microbial

374 communities help maintain beta diversity (Vannette and Fukami 2017), although we do not

375 observe an increase of beta diversity with higher dispersal, as in the experiment. Additionally,

376 Toju et al. (2018) found a persistent numeric advantage of initially abundant species after

377 prolonged dispersal in nectar microbial communities. This may indicate that dispersal in this

378 empirical study was not high enough to homogenize all local patches or priority effects

379 initiated by numeric advantage were maintained in other trait-mediated ways, such as habitat

380 modification. 


\section{Empirical Implications and Future Directions}

383 Phenology and priority effects are key factors that structure population dynamics and

384 coexistence patterns in natural communities. Although the importance of priority effects is

385 increasingly recognized (Fukami 2015; Rudolf 2019), they are rarely studied in an explicitly

386 spatial context. Our model indicates the need to consider specific mechanisms of priority

387 effects when studying spatial dynamics of metacommunities and vice versa. For instance,

388 systems that display robust diversity measurements regardless of dispersal might be more

389 influenced by trait-mediated processes. In these systems, considering specific trait-mediated

390 mechanisms of priority effects may help to understand otherwise unexpected patterns (e.g. Pu

391 and Jiang 2015; Vannette and Fukami 2017; Toju et al. 2018). Conversely, metacommunities

392 with species known to display trait-mediated priority effects (e.g. amphibians, Rudolf 2018;

393 dragonflies, Rasmussen et al. 2014; plant communities, Kardol et al. 2013; Blackford et al.

394 2020) may show different dynamics at a regional scale compared to metacommunities with

numeric priority effects. Yet, few studies have considered this aspect when studying dispersal-

396 diversity relationships in these systems, although many metacommunities are driven by

397 stochastic processes that promote priority effects (e.g. Chase 2007).

398 Our model predicts that seasonal metacommunities under spatial and temporal variations

399 in phenology may behave differently based on the mechanism of priority effects. This is

400 relevant to many systems in nature, including ephemeral ponds, nectar microbiome, pitcher

401 plant inquilines, rocky intertidal pools, and any other periodic communities where such

402 variations could occur (Berlow and Navarrete 1997; Kneitel and Miller 2003; Chase 2007;

403 Sheriff et al. 2011; Theobald et al. 2017; Vannette and Fukami 2017). Although recent studies

404 of these systems indicate that phenology can vary considerably between communities (Sheriff 
406 local and regional level consequences of these variations. As climate change exacerbates

407 phenological variation (Parmesan 2006; Diez et al. 2012; Wolkovich et al. 2014; Pearse et al.

408 2017), the arrival order of species may differ greatly over space and time, leading to increasing

409 opportunities for priority effects. Understanding how metacommunities respond to this

410 increasing reshuffling of arrival order (and likely competitive hierarchy) in the long term could

411 help evaluate responses of biodiversity to climate change.

We have extensively investigated different forms of dispersal and incorporated variations

413 in phenology, but the influence of another aspect of spatial communities, environmental

414 heterogeneity, remains unexplored. Heterogeneous patches could serve as a spatial refuge for

415 some species, promoting regional coexistence (Shurin et al. 2004). Environmental

416 heterogeneity may also induce trait-mediated priority effects, leading to monopolization effects

417 (Urban and De Meester 2009; De Meester et al. 2016) or legacy effects (Miller and Allesina

418 2021) in local patches. Furthermore, environmental heterogeneity could covary with other

419 factors that are coupled with another process, such as spatial variation of arrival time (e.g.,

420 species may arrive early in warmer patches), leading to more complex dynamics. Collectively,

421 our results emphasize that the temporal sequence of community assembly is a critical process

422 shaping biodiversity in spatial communities. Mechanistic understanding of this process is

423 fundamental to our knowledge of how biodiversity responds to critical environmental

424 processes in a changing world. 


\section{Acknowledgments}

427 We thank members of Miller and Rudolf labs for their feedback on the project and Lydia

428 Beaudrot, Joshua Fowler, Tom E. X. Miller, and Chuliang Song for commenting on the

429 manuscript. Funding was provided by NSF DEB-1655626. 


\section{$430 \quad$ Figure Captions}

431 Figure 1. Basic processes of the metacommunity model. In the hypothetical metacommunity

432 each patch is occupied by one species and the season starts when species emerge in patches

433 (step 1). Emergence time can vary across seasons and patches within a given species-specific

434 range. After emergence, species compete locally (step 2) according to competition coefficients

435 that either: (A) do not depend on the relative time of emergence (scenarios with numeric

436 priority effects), or (B) depend on the pairwise difference in relative time of emergence $(\Delta p)$

437 (scenarios with trait-mediated priority effects) and determine subsequent population dynamics

438 in a given patch. These local patch dynamics together determine regional patterns (step 3) at

439 the end of the season. Finally, the season ends with dispersal (step 4) and end-of-season

440 disturbance.

441

442 Figure 2. Example of population dynamics of one randomly selected patch under Scenario 1

443 (each patch is initially occupied by a single species with the same starting population density)

444 with different dispersal rates and mechanisms of priority effects. The left column (A, C, and E)

445 shows dynamics with numeric priority effects, and the right column (B, D, and F) shows

446 dynamics with trait-mediated priority effects. Each row shows results for low (0.001), medium

447 (0.04), and high (0.1) dispersal rates $(r)$. Each line of the same color in a panel represents the

448 population dynamics of that species from one out of 50 simulations. All population densities

449 are log-transformed. All simulations were run with 40 dispersal patches $(D=40 ; 80 \%$ of all

450 patches). See Table S1 for other parameters used. 
452 Figure 3. The regional final population of all species among all 50 simulations under Scenario

453 1, with different dispersal rates and mechanisms of priority effects. The left column (A, C, and

454 E) shows dynamics with numeric priority effects, and the right column (B, D, and F) shows

455 dynamics with trait-mediated priority effects. Each row shows results with low (0.001),

456 medium (0.04), and high (0.1) dispersal rates $(r)$. All population densities are log-transformed.

457 All simulations were run with 40 dispersal patches $(D=40 ; 80 \%$ of all patches). See Table S1

458 for other parameters used.

459

460 Figure 4. Relationship of alpha diversity (local species richness) and dispersal in

461 metacommunities with numeric vs. trait-mediated priority effects for three scenarios that differ

462 in initial conditions. Axes are parameters that determine dispersal; the $\mathrm{x}$-axis is dispersal

463 patches $(D)$, shown in percentage of overall patches $(P=50)$, and the $y$-axis is dispersal rate $(r)$.

464 The color of the shaded region indicates local richness averaged among all patches and 50

465 simulations. The left column (A, C, and E) shows dynamics with numeric priority effects, and

466 the right column (B, D, and F) shows dynamics with trait-mediated priority effects. Rows show

467 differences in initial conditions of species distributions: A-B, Scenario 1 (one species per

468 patch); C-D, Scenario 2 (equal abundance of species in all patches); E-F, Scenario 3 (different

469 species abundances across patches). For specific distribution of alpha diversity values over

470 dispersal patches, see Figure S6-8, panels A-C. See Table S1 for other parameters used.

472 Figure 5. Relationship of beta diversity (dissimilarity between patches) and dispersal in

473 metacommunities with numeric vs. trait-mediated priority effects for 3 scenarios that differ in

474 initial conditions. Axes are parameters that determine dispersal; the $\mathrm{x}$-axis is dispersal patches 
$475(D)$, shown in percentage of overall patches $(P=50)$ and the $y$-axis is dispersal rate $(r)$. The

476 color of the shaded region indicates dissimilarity averaged among 50 simulations. The left

477 column (A, C, and E) shows dynamics with numeric priority effects, and the right column (B,

478 D, and F) shows dynamics with trait-mediated priority effects. Rows show differences in initial

479 conditions of species distributions: A-B, Scenario 1 (one species per patch); C-D, Scenario 2

480 (equal abundance of species in all patches); E-F, Scenario 3 (different species abundances

481 across patches). For specific distribution of alpha diversity values over dispersal patches, see

482 Figure S6-8, panels D-F. See Table S1 for other parameters used.

483

484 Figure 6. Temporal turnover calculated from one randomly selected simulation under Scenario

4851 with different dispersal rates and mechanisms of priority effects. The left column (A, C, and

486 E) shows dynamics with numeric priority effects, and the right column (B, D, and F) shows

487 dynamics with trait-mediated priority effects. Rows show results with low (0.001), medium

488 (0.04) and high (0.1) dispersal rates $(r)$. The number of patches with dispersal $(D)$ is shown as a

489 percentage of total patches and represented by different shades of color. The dissimilarity index

490 was calculated from metacommunities between consecutive time steps separated by 10 seasons. 


\section{Reference}

492

493

494

495

496

497

498

499

500

501

502

503

504

505

506

507

508

509

510

511

512

513

514

515

516

517

518

519

520

521

522

523

524

525

526

527

528

529

530

531

532

533

534

535
Alford, R. A., and H. M. Wilbur. 1985. "Priority Effects in Experimental Pond Communities: Competition between Bufo and Rana." Ecology. https://doi.org/10.2307/1939161.

Allesina, Stefano, and Jonathan M. Levine. 2011. "A Competitive Network Theory of Species Diversity." Proceedings of the National Academy of Sciences of the United States of America 108 (14): 5638-42. https://doi.org/10.1073/pnas.1014428108.

Altermatt, F, S Schreiber, and M Holyoak. 2011. "Interactive Effects of Disturbance and Dispersal Directionality on Species Richness and Composition in Metacommunities." Ecology. https://doi.org/10.1890/10-1095.1.

Berlow, Eric L., and Sergio A. Navarrete. 1997. "Spatial and Temporal Variation in Rocky Intertidal Community Organization: Lessons from Repeating Field Experiments." Journal of Experimental Marine Biology and Ecology 214 (1-2): 195-229. https://doi.org/10.1016/S0022-0981(97)00023-3.

Blackford, Christopher, Rachel M Germain, and Benjamin Gilbert. 2020. "Species Differences in Phenology Shape Coexistence." The American Naturalist 195 (6). https://doi.org/10.1086/708719.

Cadotte, Marc W. 2006. "Dispersal and Species Diversity: A Meta-Analysis." American Naturalist. https://doi.org/10.1086/504850.

Carrara, Francesco, Florian Altermatt, Ignacio Rodriguez-Iturbe, and Andrea Rinaldo. 2012. "Dendritic Connectivity Controls Biodiversity Patterns in Experimental Metacommunities." Proceedings of the National Academy of Sciences.

Carter, Shannon K., Daniel Saenz, and Volker H.W. Rudolf. 2018. "Shifts in Phenological Distributions Reshape Interaction Potential in Natural Communities." Ecology Letters 21 (8): 1143-51. https://doi.org/10.1111/ele.13081.

Catano, Christopher P., Timothy L. Dickson, and Jonathan A. Myers. 2017. "Dispersal and Neutral Sampling Mediate Contingent Effects of Disturbance on Plant Beta-Diversity: A Meta-Analysis." Ecology Letters 20 (3): 347-56. https://doi.org/10.1111/ele.12733.

Chase, Jonathan M. 2007. "Drought Mediates the Importance of Stochastic Community Assembly." Proceedings of the National Academy of Sciences of the United States of America. https://doi.org/10.1073/pnas.0704350104.

De Meester, Luc, Joost Vanoverbeke, Laurens J. Kilsdonk, and Mark C. Urban. 2016. "Evolving Perspectives on Monopolization and Priority Effects." Trends in Ecology and Evolution 31 (2): 136-46. https://doi.org/10.1016/j.tree.2015.12.009.

DeMalach, Niv, Nadav Shnerb, and Tadashi Fukami. 2021. "Alternative States in Plant Communities Driven by a Life-History Trade-Off and Demographic Stochasticity." The American Naturalist, March, E000-E000. https://doi.org/10.1086/714418.

Diez, Jeffrey M., Inés Ibáñez, Abraham J. Miller-Rushing, Susan J. Mazer, Theresa M. Crimmins, Michael A. Crimmins, C. David Bertelsen, and David W. Inouye. 2012. "Forecasting Phenology: From Species Variability to Community Patterns." Ecology Letters. https://doi.org/10.1111/j.1461-0248.2012.01765.x.

Drake, J. A. 1991. "Community-Assembly Mechanics and the Structure of an Experimental Species Ensemble." American Naturalist 137 (1): 1-26. https://doi.org/10.1086/285143.

Dray, Stéphane, Guillaume Blanchet, Daniel Borcard, Guillaume Guenard, Thibaut Jombart, Guillaume Larocque, Pierre Legendre, Naima Madi, Helene H Wagner, and Maintainer Stéphane Dray. 2018. "Package 'Adespatial."' $R$ Package Version, 3-8. 
Fodelianakis, Stilianos, Alexander Lorz, Adriana Valenzuela-Cuevas, Alan Barozzi, Jenny Marie Booth, and Daniele Daffonchio. 2019. "Dispersal Homogenizes Communities via Immigration Even at Low Rates in a Simplified Synthetic Bacterial Metacommunity." Nature Communications 10 (1): 1314. https://doi.org/10.1038/s41467-019-09306-7.

Fukami, Tadashi. 2015. "Historical Contingency in Community Assembly: Integrating Niches, Species Pools, and Priority Effects." Annual Review of Ecology, Evolution, and Systematics 46: 1-23.

Grainger, Tess Nahanni, and Benjamin Gilbert. 2016. "Dispersal and Diversity in Experimental Metacommunities: Linking Theory and Practice." Oikos 125 (9): 1213-23. https://doi.org/10.1111/oik.03018.

Howeth, Jennifer G., and Mathew A. Leibold. 2010a. "Prey Dispersal Rate Affects Prey Species Composition and Trait Diversity in Response to Multiple Predators in Metacommunities." Journal of Animal Ecology 79 (5): 1000-1011. https://doi.org/10.1111/j.1365-2656.2010.01715.x. 2010b. "Species Dispersal Rates Alter Diversity and Ecosystem Stability in Pond Metacommunities." Ecology 91 (9): 2727-41. https://doi.org/10.1890/09-1004.1.

Kardol, Paul, Lara Souza, and Aimée T Classen. 2013. "Resource Availability Mediates the Importance of Priority Effects in Plant Community Assembly and Ecosystem Function." Oikos.

Ke, Po Ju, and Andrew D. Letten. 2018. "Coexistence Theory and the Frequency-Dependence of Priority Effects." Nature Ecology and Evolution 2 (11): 1691-95. https://doi.org/10.1038/s41559-018-0679-z.

Kerr, B, M A Riley, M W Feldman, and B J M Bohannan. 2002. "Local Dispersal Promotes Biodiversity in a Real-Life Game of Rock-Paper-Scissors." Nature. https://doi.org/10.1038/nature00823.

Kneitel, Jamie M, and Thomas E Miller. 2003. "Dispersal Rates Affect Species Composition in Metacommunities of Sarracenia Purpurea Inquilines." American Naturalist 162 (2): $165-71$.

Legendre, Pierre. 2019. "A Temporal Beta $\square$ diversity Index to Identify Sites That Have Changed in Exceptional Ways in Space-Time Surveys." Ecology and Evolution 9 (6): 3500-3514. https://doi.org/10.1002/ece3.4984.

Leibold, M. A., M. Holyoak, N. Mouquet, P. Amarasekare, J. M. Chase, M. F. Hoopes, R. D. Holt, et al. 2004. "The Metacommunity Concept: A Framework for Multi-Scale Community Ecology." Ecology Letters. https://doi.org/10.1111/j.14610248.2004.00608.x.

Leibold, Mathew A., Mark C. Urban, Luc De Meester, Christopher A. Klausmeier, and Joost Vanoverbeke. 2019. "Regional Neutrality Evolves through Local Adaptive Niche Evolution." Proceedings of the National Academy of Sciences of the United States of America 116 (7): 2612-17. https://doi.org/10.1073/pnas.1808615116.

Levine, Jonathan M., Jordi Bascompte, Peter B. Adler, and Stefano Allesina. 2017. "Beyond Pairwise Mechanisms of Species Coexistence in Complex Communities." Nature 546 (7656): 56-64. https://doi.org/10.1038/nature22898.

Lu, Muyang. 2021. "Complex Relationships between Beta Diversity and Dispersal in MetaCommunity Models.” Ecography n/a (n/a). https://doi.org/10.1111/ecog.05937.

Miller, Zachary R., and Stefano Allesina. 2021. "Metapopulations with Habitat Modification." Proceedings of the National Academy of Sciences 118

(49). 
https://doi.org/10.1073/pnas.2109896118.

Mouquet, Nicolas, and Michel Loreau. 2002. "Coexistence in Metacommunities: The Regional Similarity Hypothesis." American Naturalist $159 \quad$ (4): 420-26. https://doi.org/10.1086/338996. . 2003. "Community Patterns in Source-Sink Metacommunities." American Naturalist. https://doi.org/10.1086/378857.

Oksanen, Jari, F Guillaume Blanchet, Roeland Kindt, Pierre Legendre, Peter R Minchin, RB O'hara, Gavin L Simpson, et al. 2013. "Package "Vegan." Community Ecology Package, Version 2 (9): 1-295.

Parmesan, Camille. 2006. "Ecological and Evolutionary Responses to Recent Climate Change." Annual Review of Ecology, Evolution, and Systematics. https://doi.org/10.1146/annurev.ecolsys.37.091305.110100.

Pearse, William D., Charles C. Davis, David W. Inouye, Richard B. Primack, and T. Jonathan Davies. 2017. "A Statistical Estimator for Determining the Limits of Contemporary and Historic Phenology." Nature Ecology and Evolution 1 (12): 1876-82. https://doi.org/10.1038/s41559-017-0350-0.

Poulos, Davina E., and Mark I. McCormick. 2014. "Who Wins in the Battle for Space? The Importance of Priority, Behavioural History and Size." Animal Behaviour 90: 305-14. https://doi.org/10.1016/j.anbehav.2014.02.003.

$\mathrm{Pu}$, Zhichao, and Lin Jiang. 2015. "Dispersal among Local Communities Does Not Reduce Historical Contingencies during Metacommunity Assembly." Oikos 124 (10): 1327-36. https://doi.org/10.1111/oik.02079.

Pulliam, H. Ronald. 1988. "Sources, Sinks, and Population Regulation." The American Naturalist 132 (5): 652-61.

R Core Team. 2021. "R: A Language and Environment for Statistical Computing."

Rasmussen, Nick L., Benjamin G. Van Allen, and Volker H.W. Rudolf. 2014. "Linking Phenological Shifts to Species Interactions through Size-Mediated Priority Effects." Journal of Animal Ecology 83 (5): 1206-15. https://doi.org/10.1111/1365-2656.12203.

Rudolf, Volker H.W. 2018. "Nonlinear Effects of Phenological Shifts Link Interannual Variation to Species Interactions." Journal of Animal Ecology 87 (5): 1395-1406. https://doi.org/10.1111/1365-2656.12850. . 2019. "The Role of Seasonal Timing and Phenological Shifts for Species Coexistence." Ecology Letters 22 (8): 1324-38. https://doi.org/10.1111/ele.13277.

Sheriff, Michael J., G. Jim Kenagy, Melanie Richter, Trixie Lee, Øivind Tøien, Franziska Kohl, C. Loren Buck, and Brian M. Barnes. 2011. "Phenological Variation in Annual Timing of Hibernation and Breeding in Nearby Populations of Arctic Ground Squirrels." Proceedings of the Royal Society B: Biological Sciences 278 (1716): 2369-75. https://doi.org/10.1098/rspb.2010.2482.

Shorrocks, Bryan, and Marc Bingley. 1994. "Priority Effects and Species Coexistence: Experiments with Fungal-Breeding Drosophila." Journal of Animal Ecology 63 (4): 799-806.

Shurin, Jonathan B., Priyanga Amarasekare, Jonathan M. Chase, Robert D. Holt, Martha F. Hoopes, and Mathew A. Leibold. 2004. "Alternative Stable States and Regional Community Structure." Journal of Theoretical Biology 227 (3): 359-68. https://doi.org/10.1016/j.jtbi.2003.11.013.

Theobald, Elli J., Ian Breckheimer, and Janneke HilleRisLambers. 2017. "Climate Drives 
Phenological Reassembly of a Mountain Wildflower Meadow Community.” Ecology 98 (11): 2799-2812. https://doi.org/10.1002/ecy.1996.

Thompson, Patrick L., Laura Melissa Guzman, Luc De Meester, Zsófia Horváth, Robert Ptacnik, Bram Vanschoenwinkel, Duarte S. Viana, and Jonathan M. Chase. 2020. "A Process-Based Metacommunity Framework Linking Local and Regional Scale Community Ecology." Ecology Letters $23 \quad$ (9): 1314-29. https://doi.org/10.1111/ele.13568.

Tilman, David. 1994. “Competition and Biodiversity in Spatially Structured Habitats.” Ecology 75 (1): 2-16. https://doi.org/10.2307/1939377.

Toju, Hirokazu, Rachel L. Vannette, Marie Pierre L. Gauthier, Manpreet K. Dhami, and Tadashi Fukami. 2018. "Priority Effects Can Persist across Floral Generations in Nectar Microbial Metacommunities." Oikos 127 (3): 345-52. https://doi.org/10.1111/oik.04243.

Urban, M C, and L De Meester. 2009. "Community Monopolization: Local Adaptation Enhances Priority Effects in an Evolving Metacommunity." Proceedings of the Royal Society B-Biological Sciences. https://doi.org/10.1098/rspb.2009.1382.

Vannette, Rachel L., and Tadashi Fukami. 2017. "Dispersal Enhances Beta Diversity in Nectar Microbes.” Ecology Letters 20 (7): 901-10. https://doi.org/10.1111/ele.12787.

Vanoverbeke, Joost, Mark C. Urban, and Luc De Meester. 2016. "Community Assembly Is a Race between Immigration and Adaptation: Eco-Evolutionary Interactions across Spatial Scales.” Ecography 39 (9): 858-70. https://doi.org/10.1111/ecog.01394.

Vanschoenwinkel, Bram, Falko Buschke, and Luc Brendonck. 2013. "Disturbance Regime Alters the Impact of Dispersal on Alpha and Beta Diversity in a Natural Metacommunity.” Ecology 94 (11): 2547-57. https://doi.org/10.1890/12-1576.1.

Wolkovich, E. M., B. I. Cook, K. K. McLauchlan, and T. J. Davies. 2014. “Temporal Ecology in the Anthropocene." Ecology Letters. https://doi.org/10.1111/ele.12353.

Zou, Heng-Xing, and Volker H. W. Rudolf. 2020. "Priority Effects and Season Length Shape Long-Term Competition Dynamics.” BioRxiv, 2020.08.14.251926. 


\section{End-of-Season Disturbance, then Next Season}

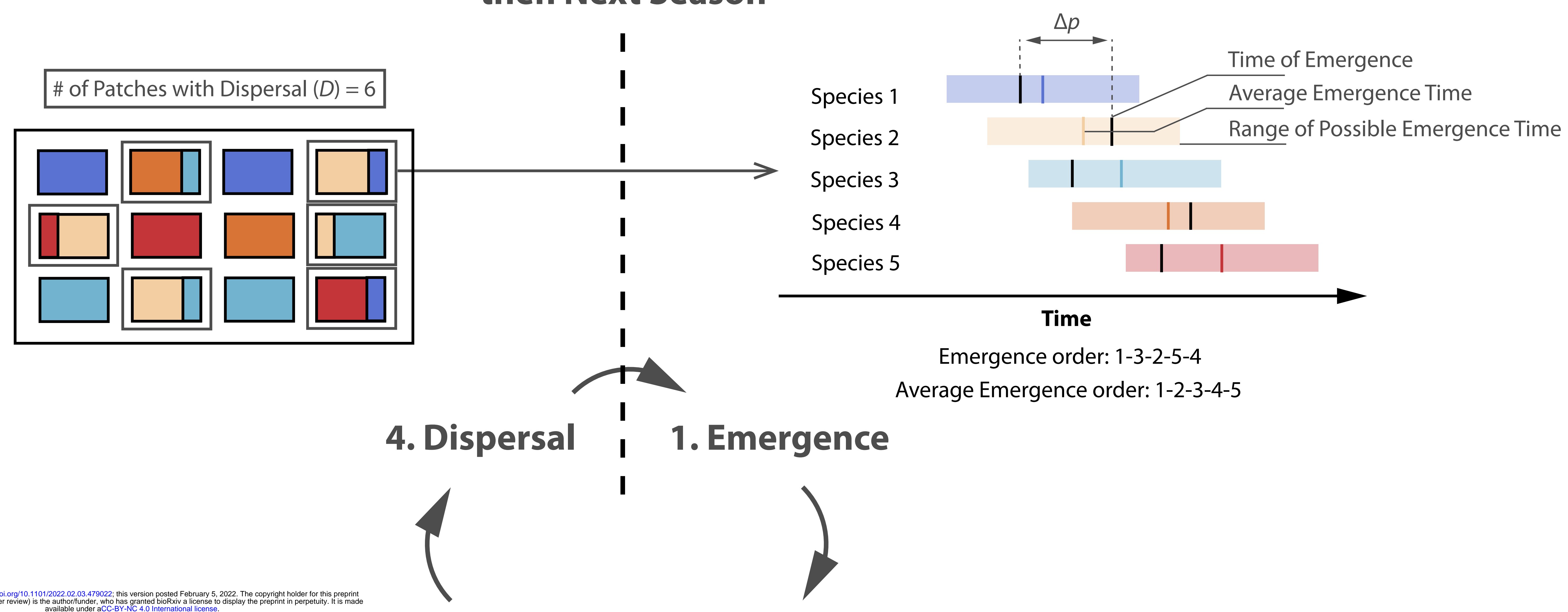

\section{Regional Pattern 2. Local Dynamics}
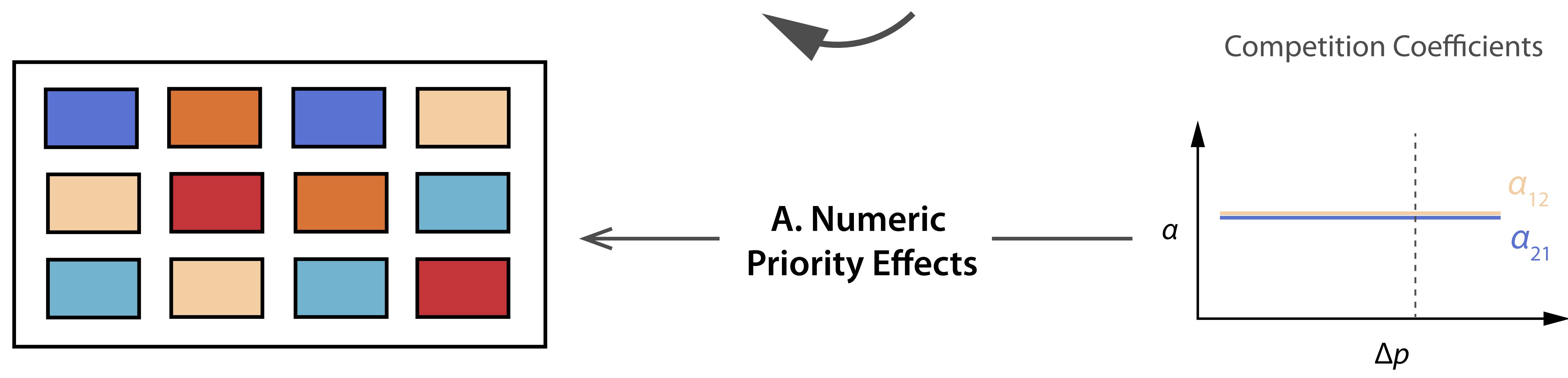

Population Dynamics

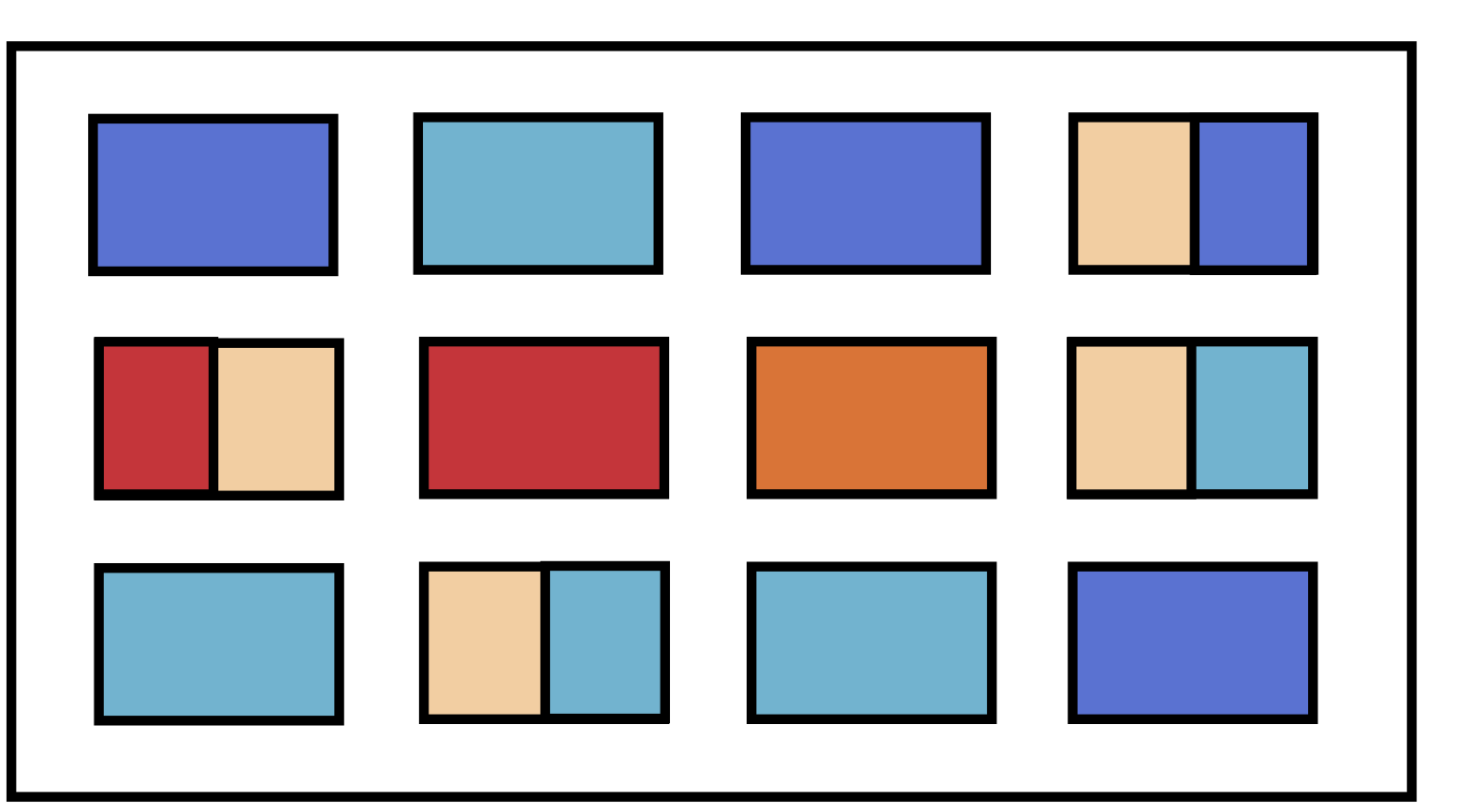

$\longleftarrow \quad$ B. Trait-mediated Priority Effects
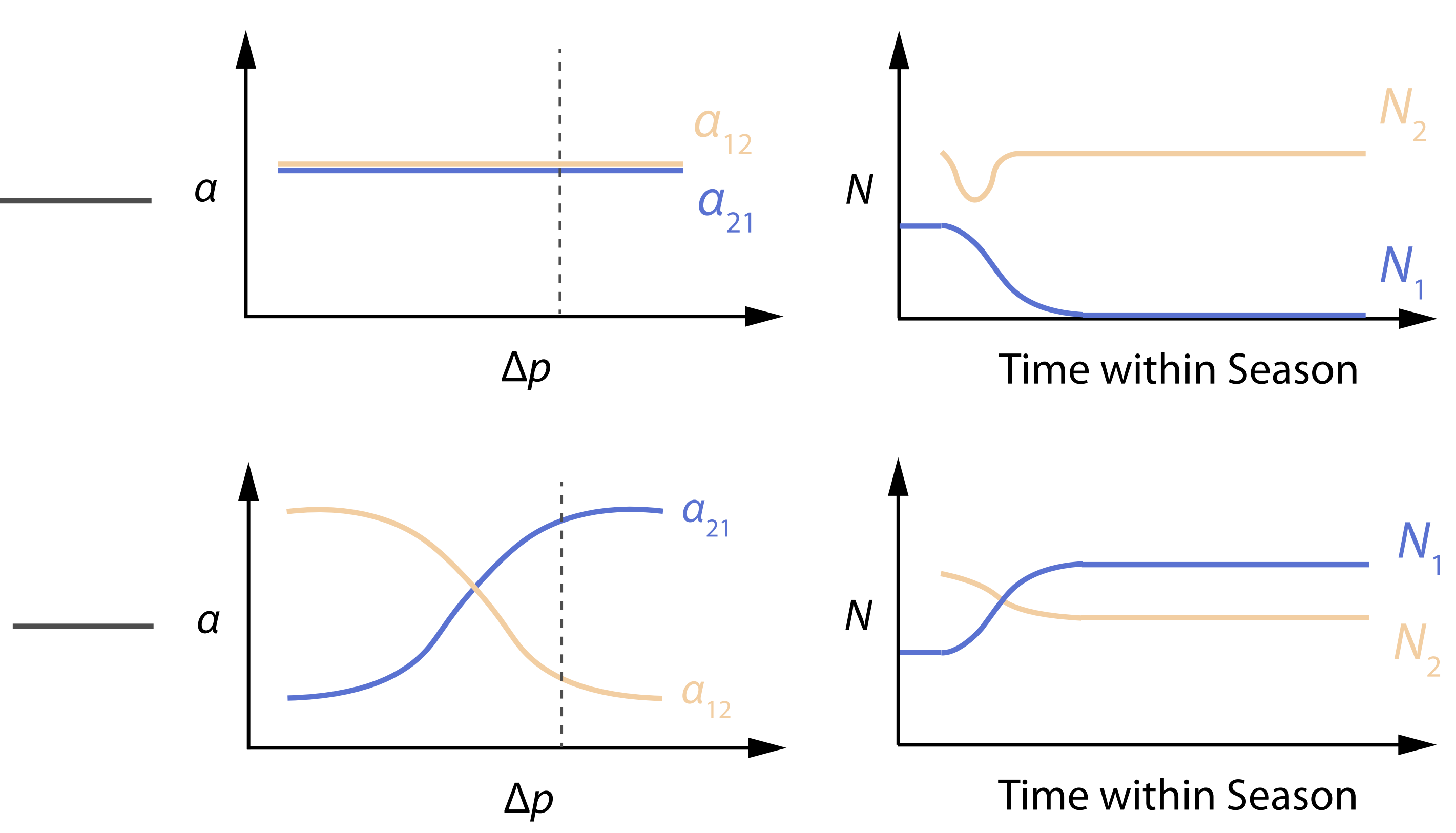
Numeric Priority Effects Trait-Mediated Priority Effects

A B
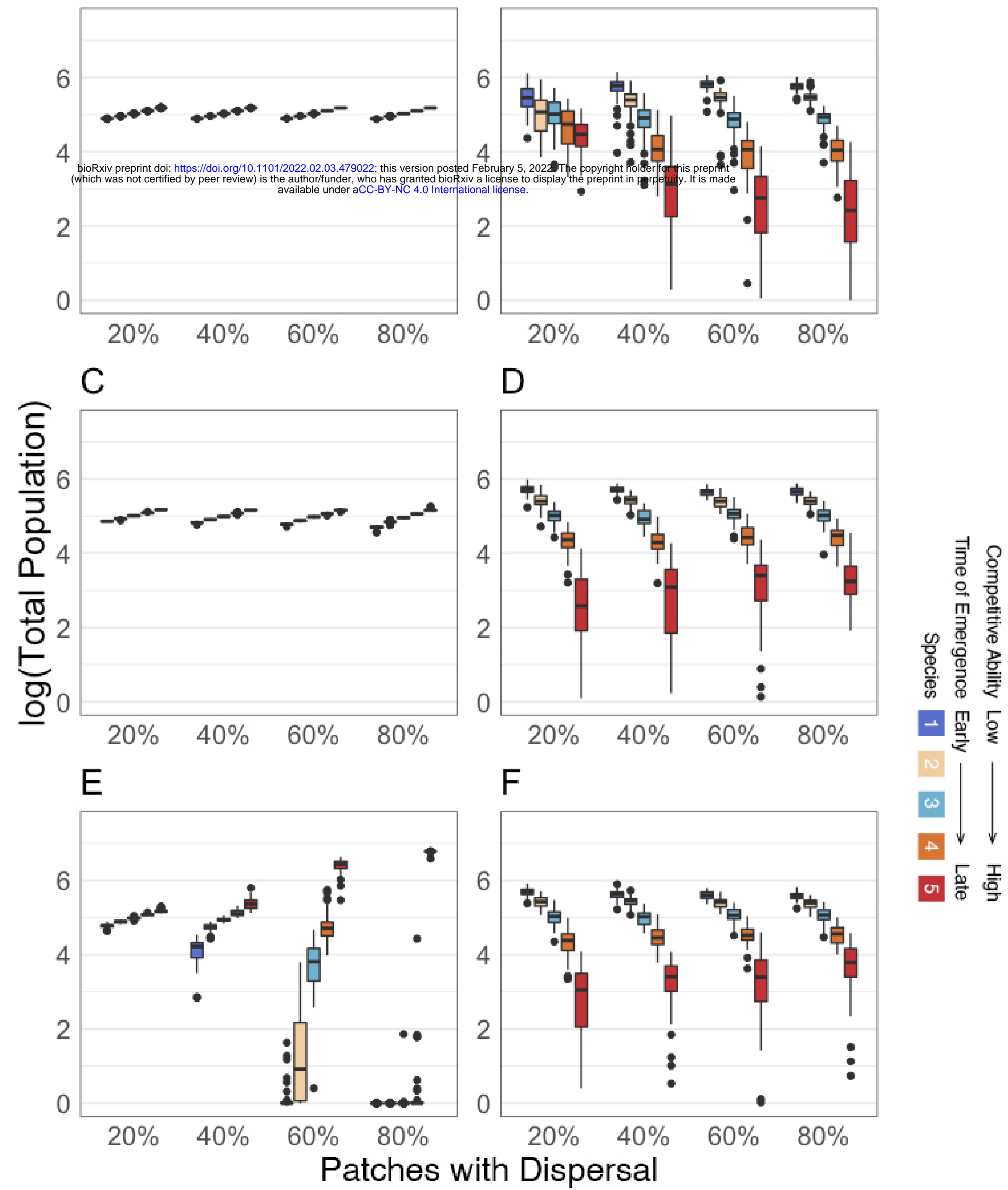


\section{Numeric Priority Effects Trait-Mediated Priority Effects}

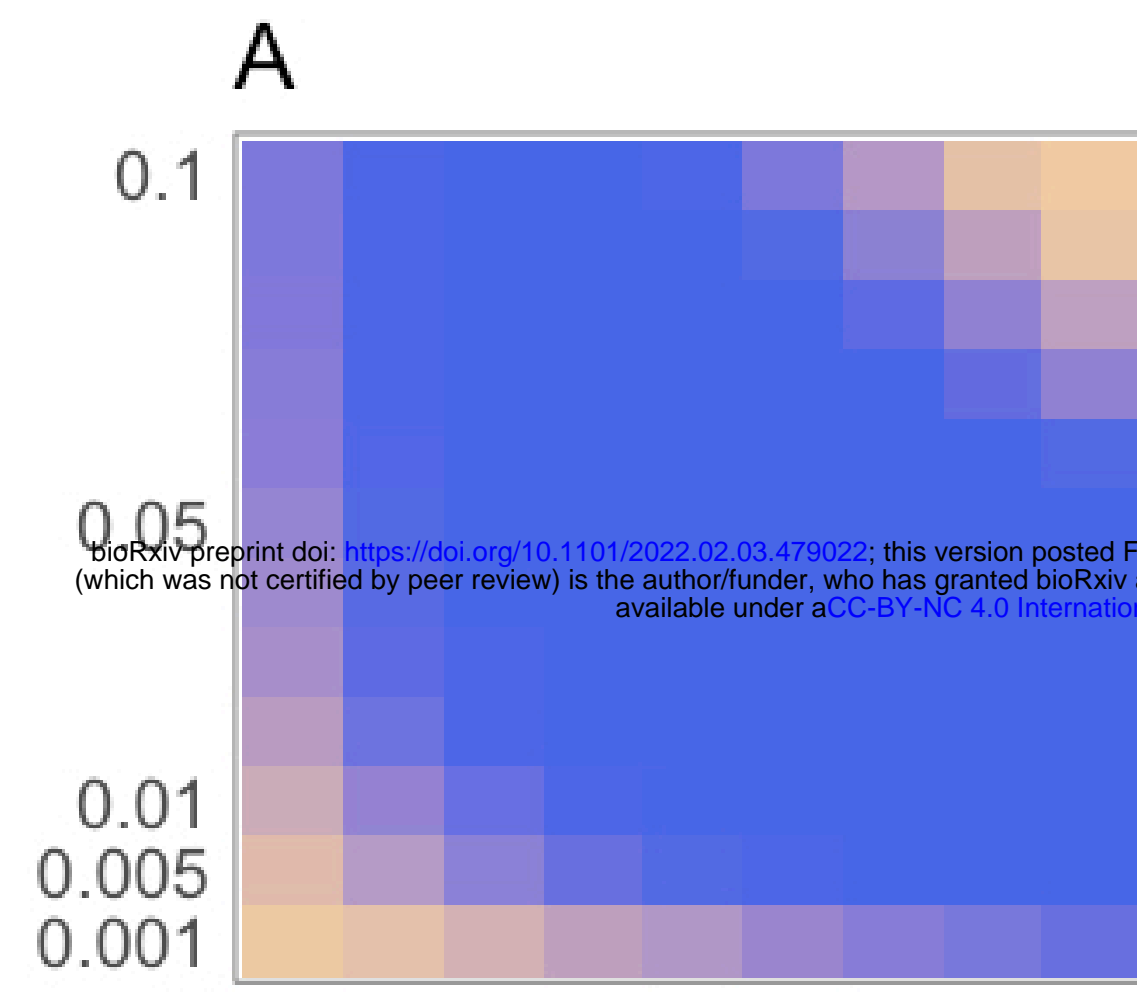

B

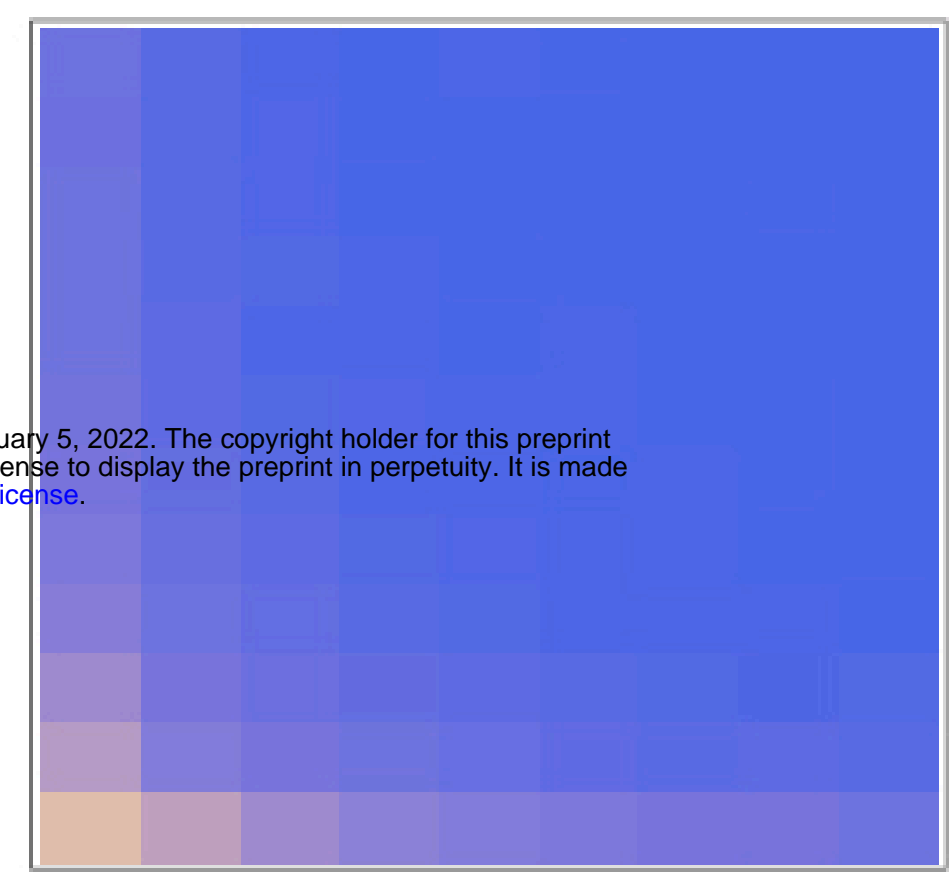

Scenario 1

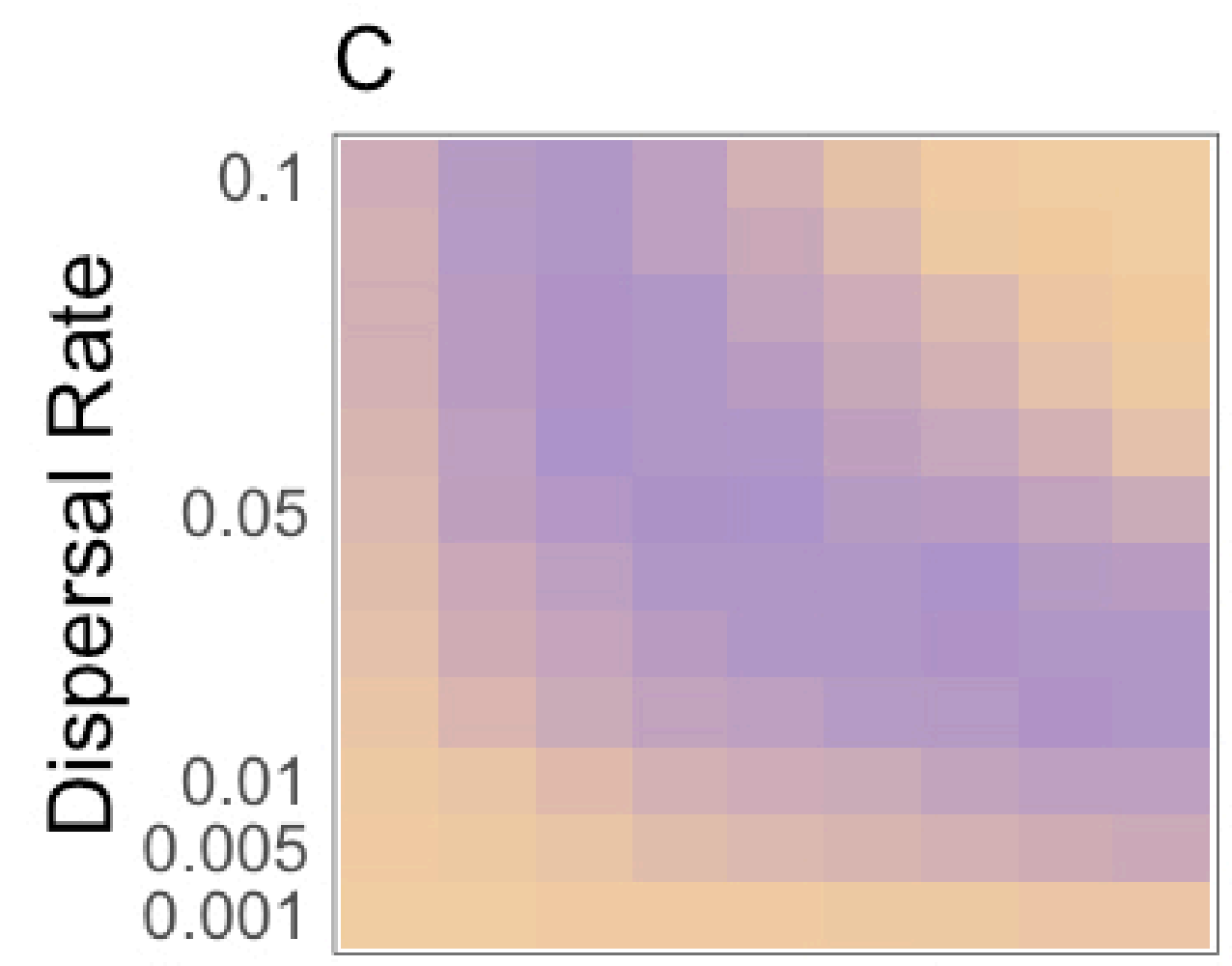

D

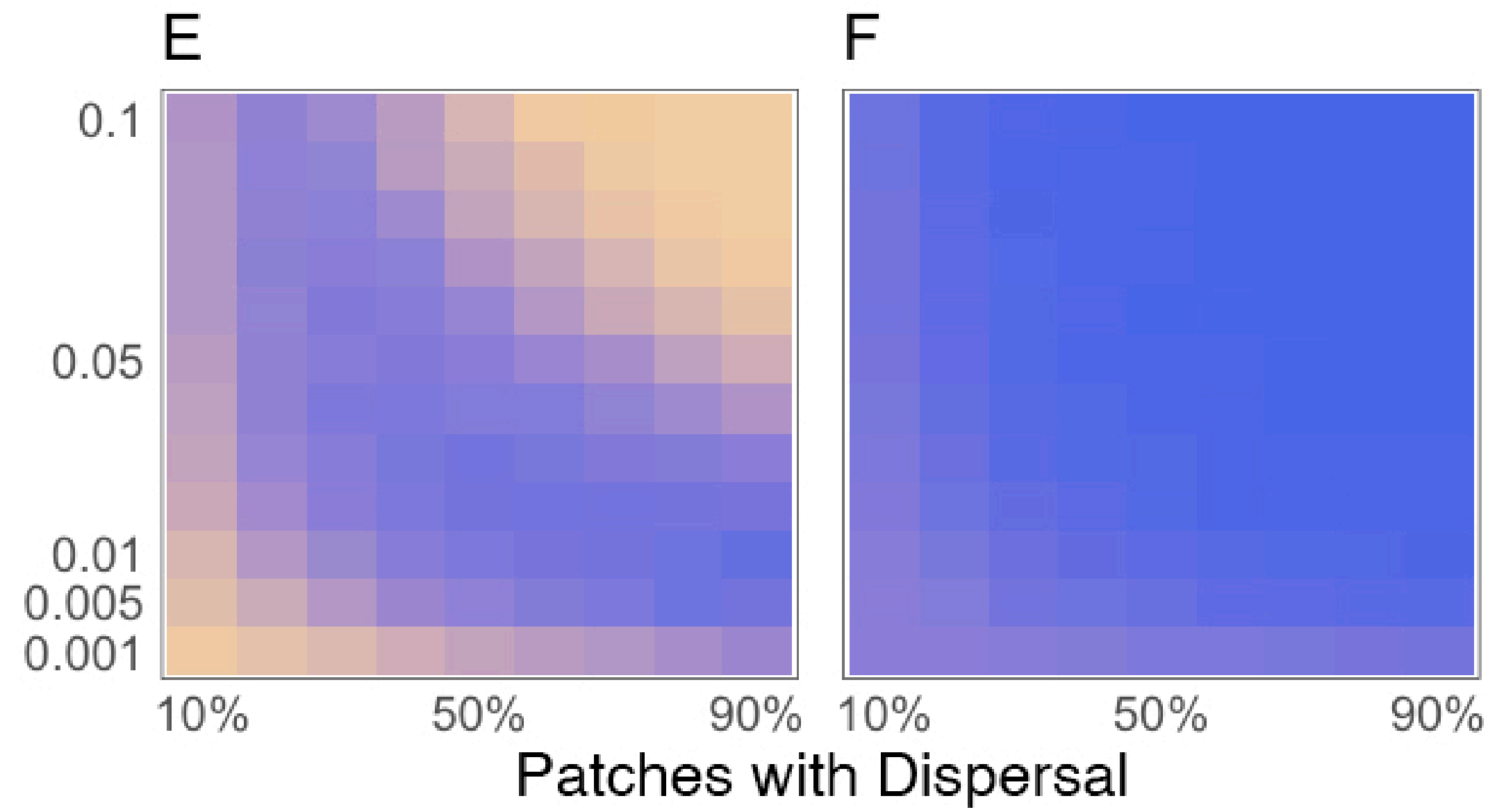

Scenario 3

Richness

5
4
3
2
1




\section{Numeric Priority Effects Trait-Mediated Priority Effects}

A B

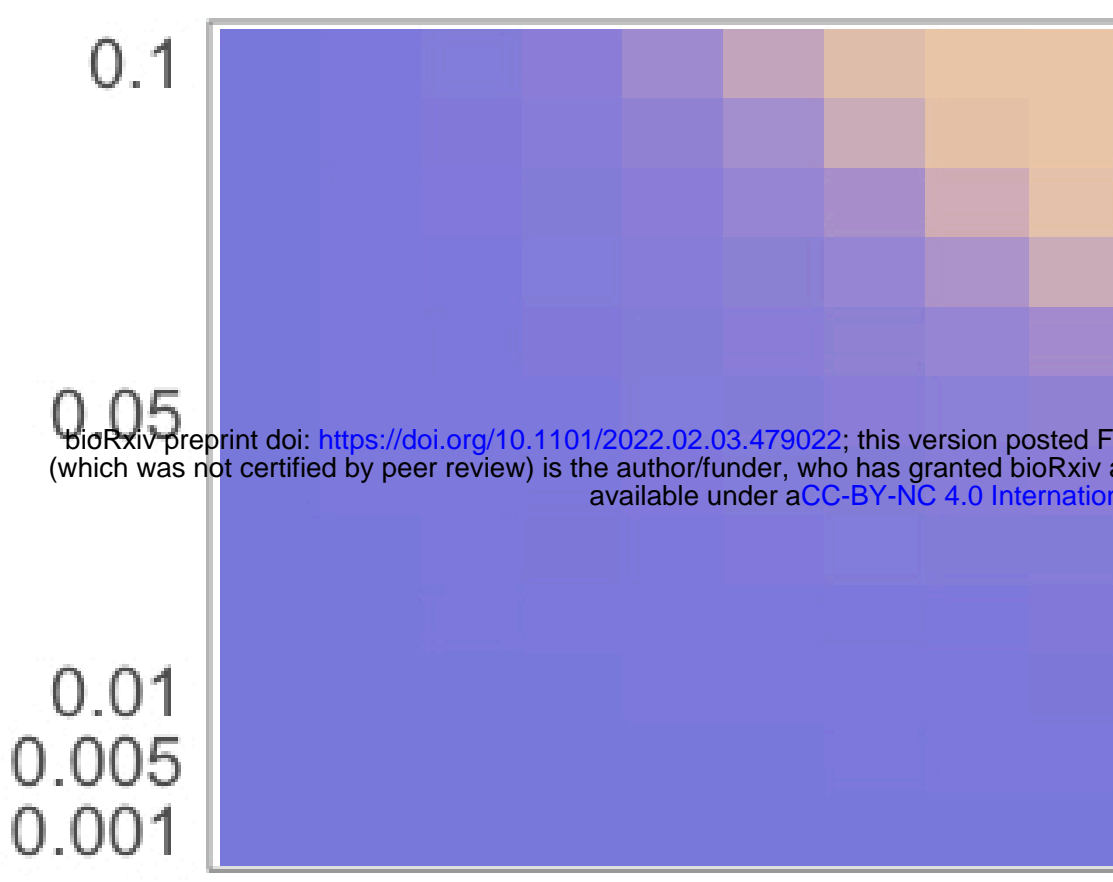

C

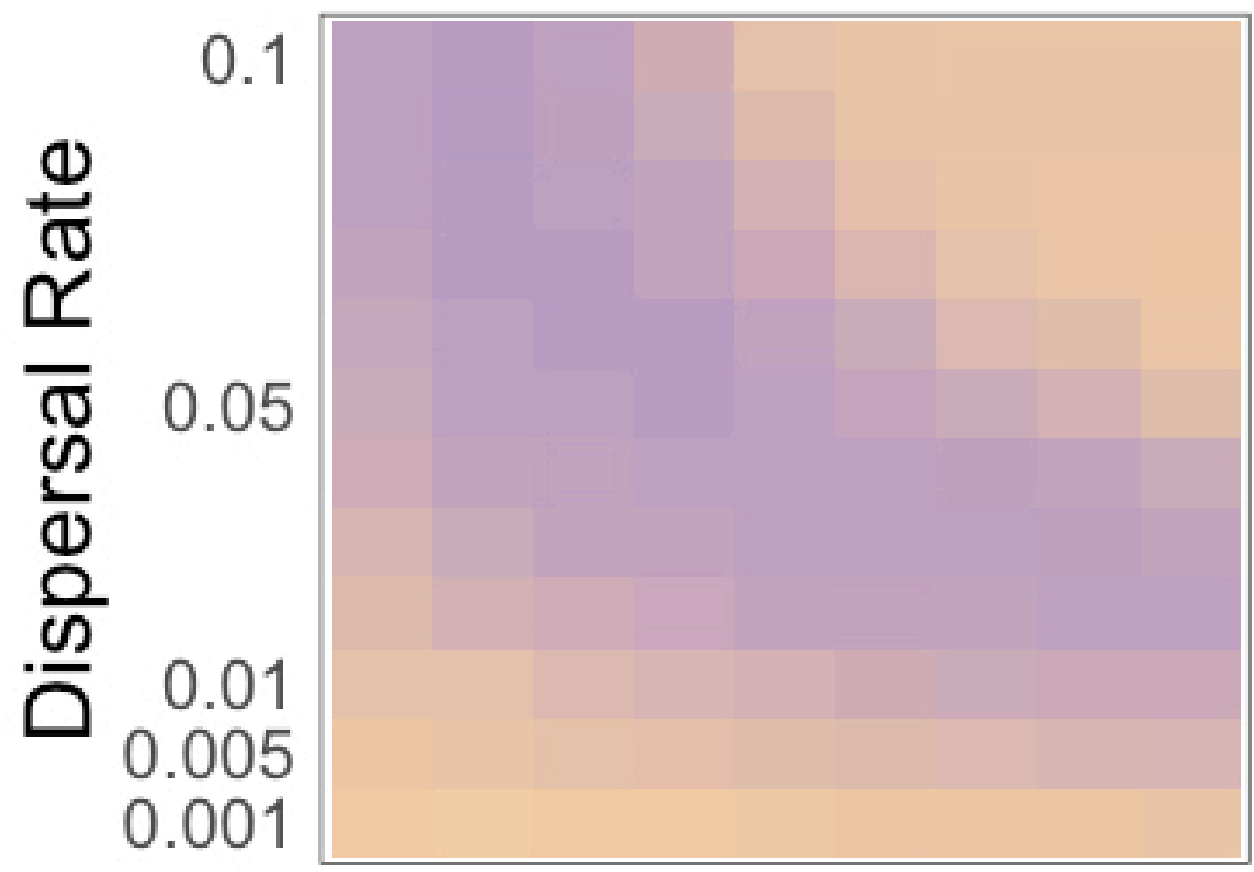

E

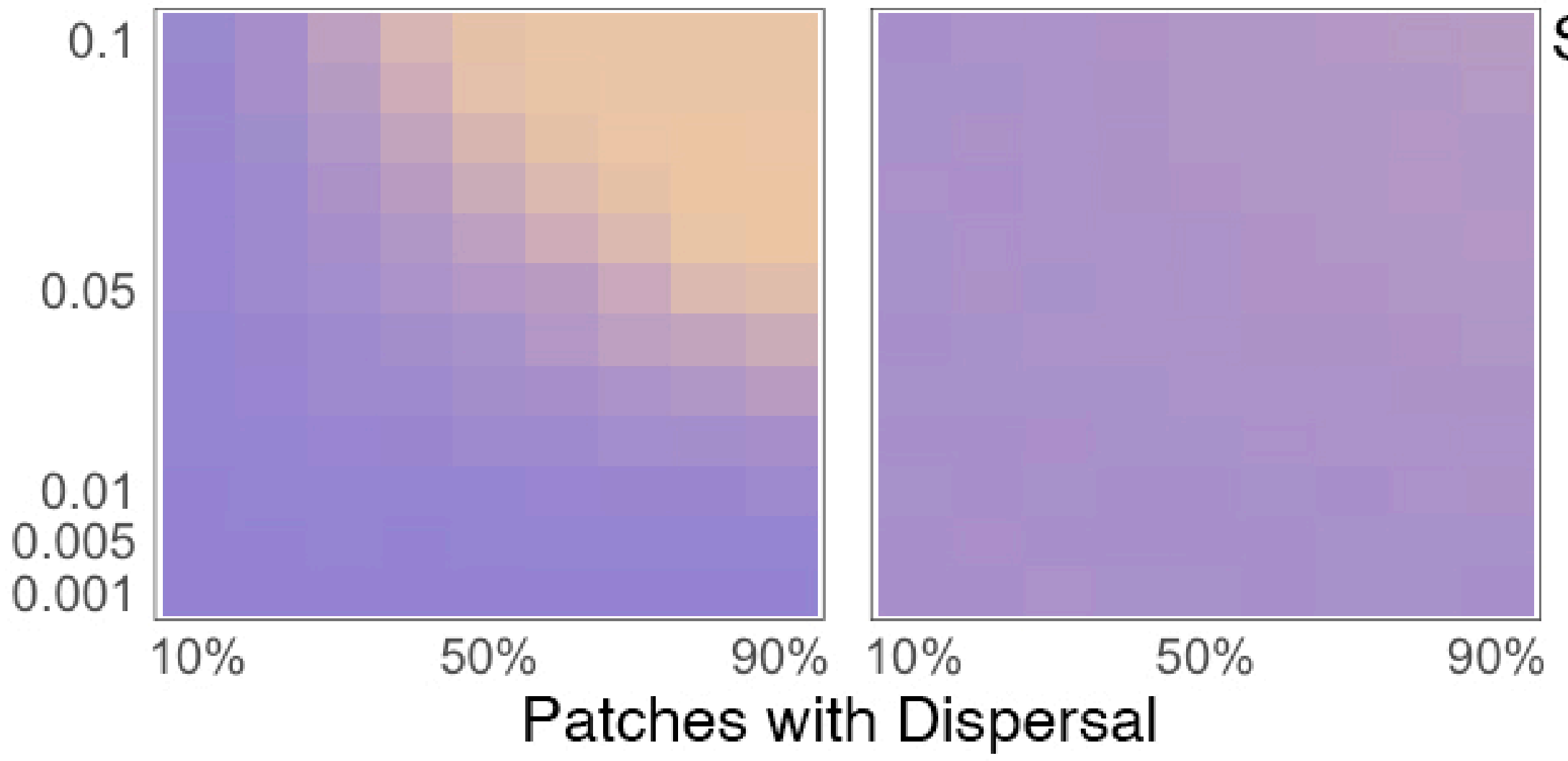

Scenario 1

Scenario 2

Scenario 3

Beta Diversity

1.00
0.75
0.50
0.25
0.00

0.75

0.50

0.00 
Numeric Priority Effects Trait-Mediated Priority Effects

A

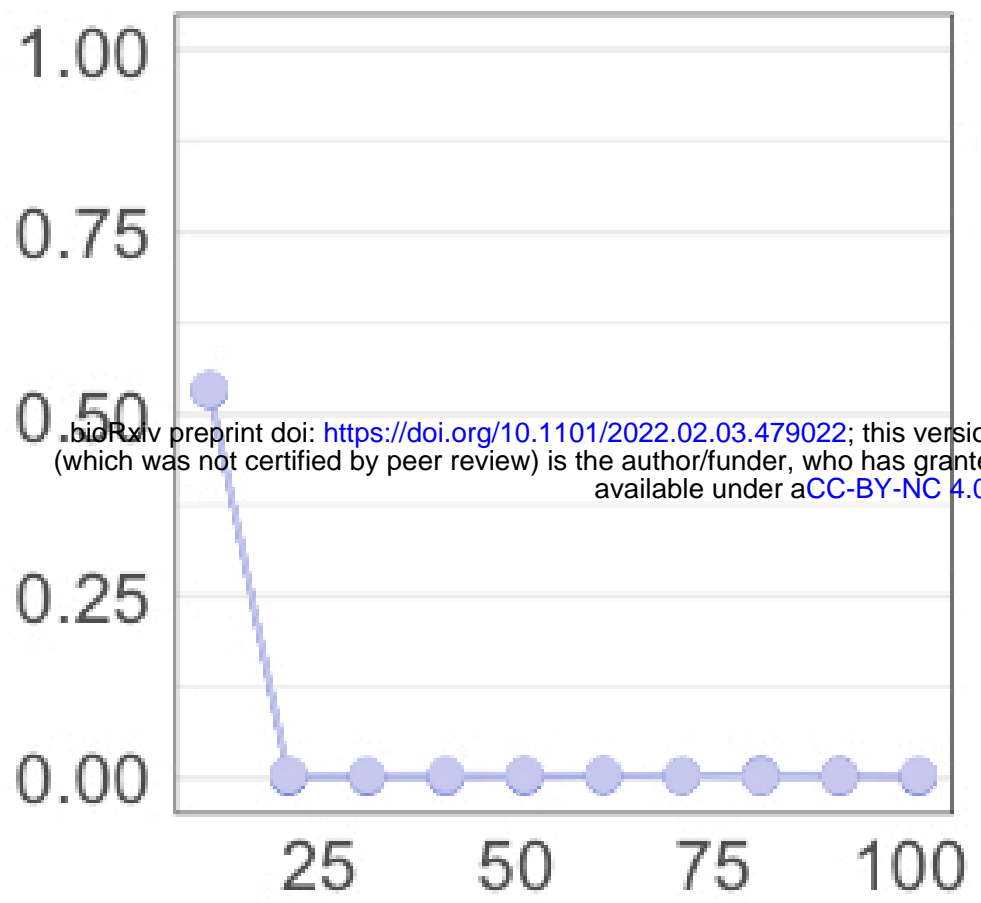

C

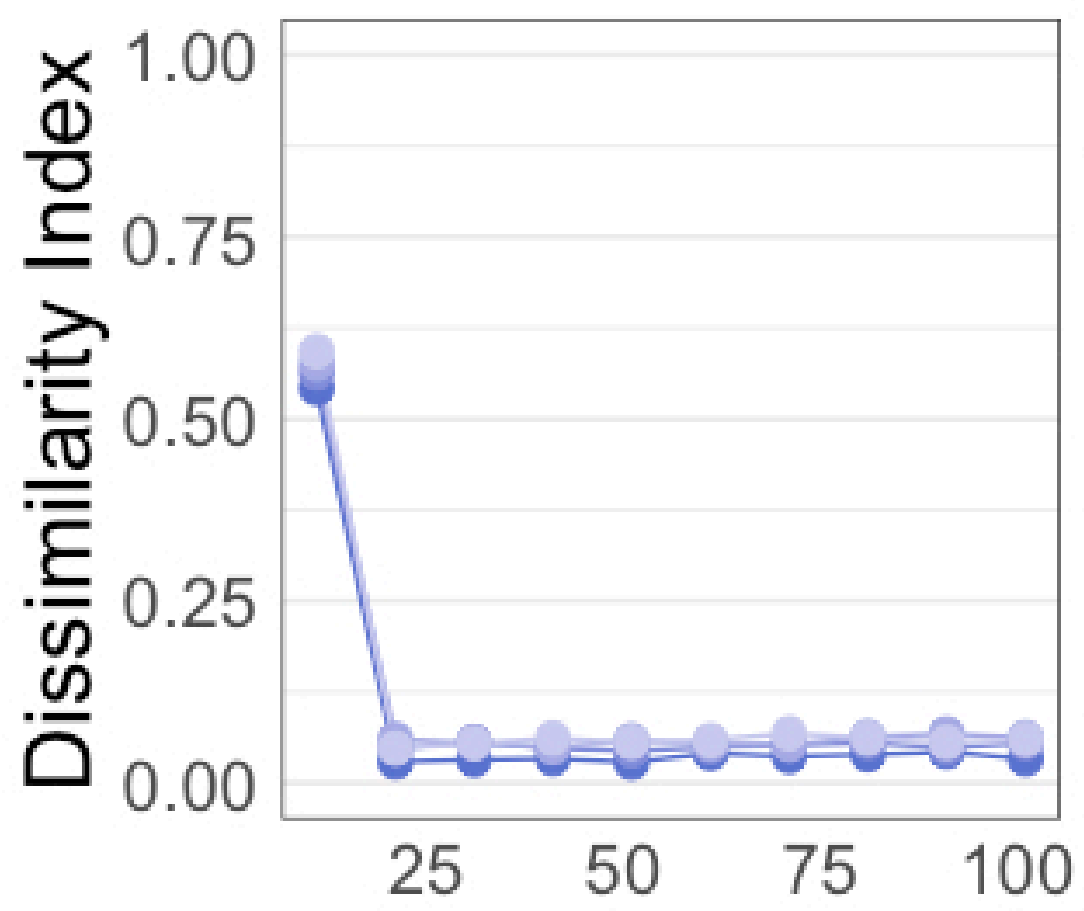

$\mathrm{E}$

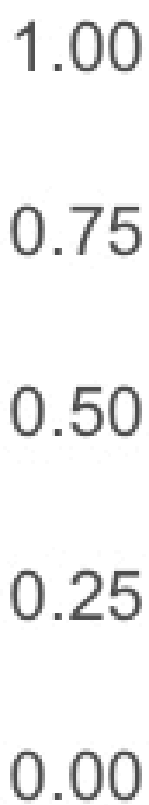

0.00

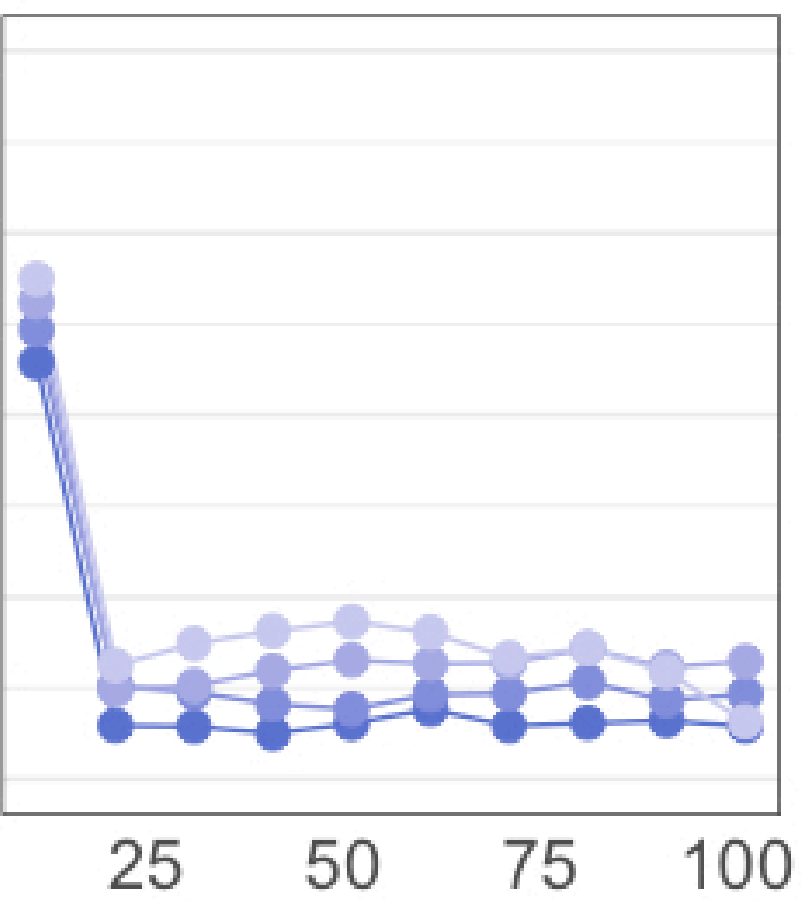

B

1.00

0.75

250

ary 5,2022 The copyright holder for this preprint

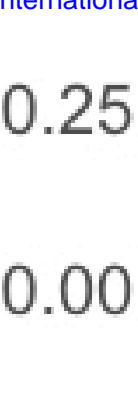

0.25

0.00

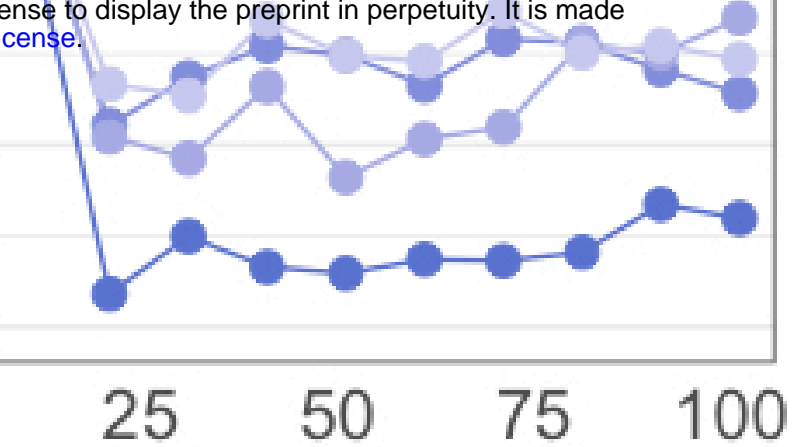

D

1.00

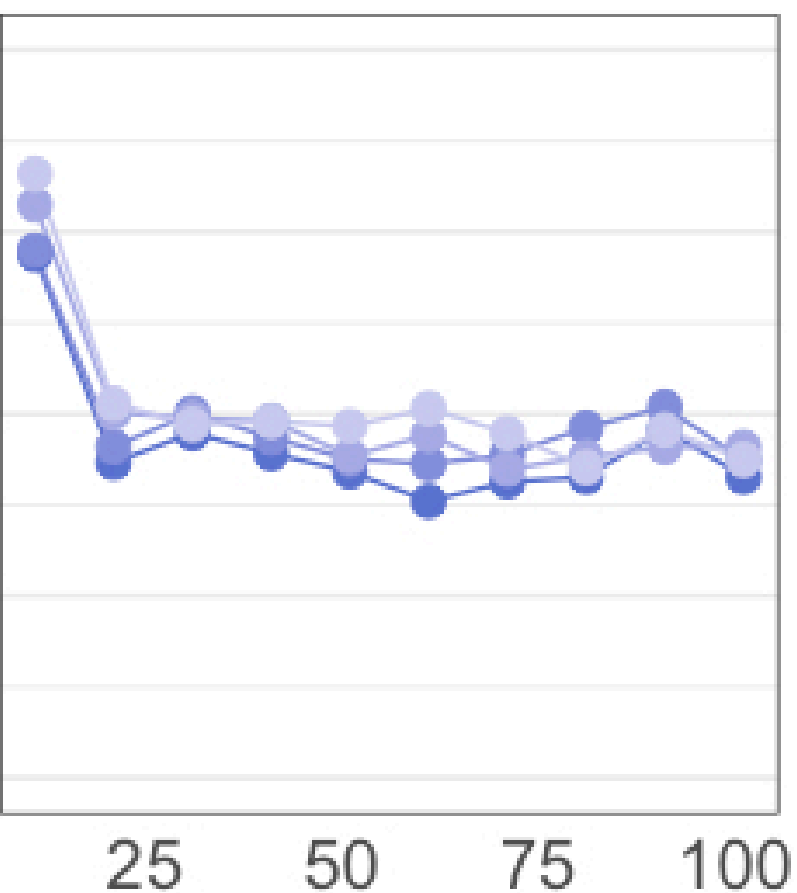

$\mathrm{F}$

1.00

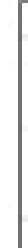

0.75

0.50

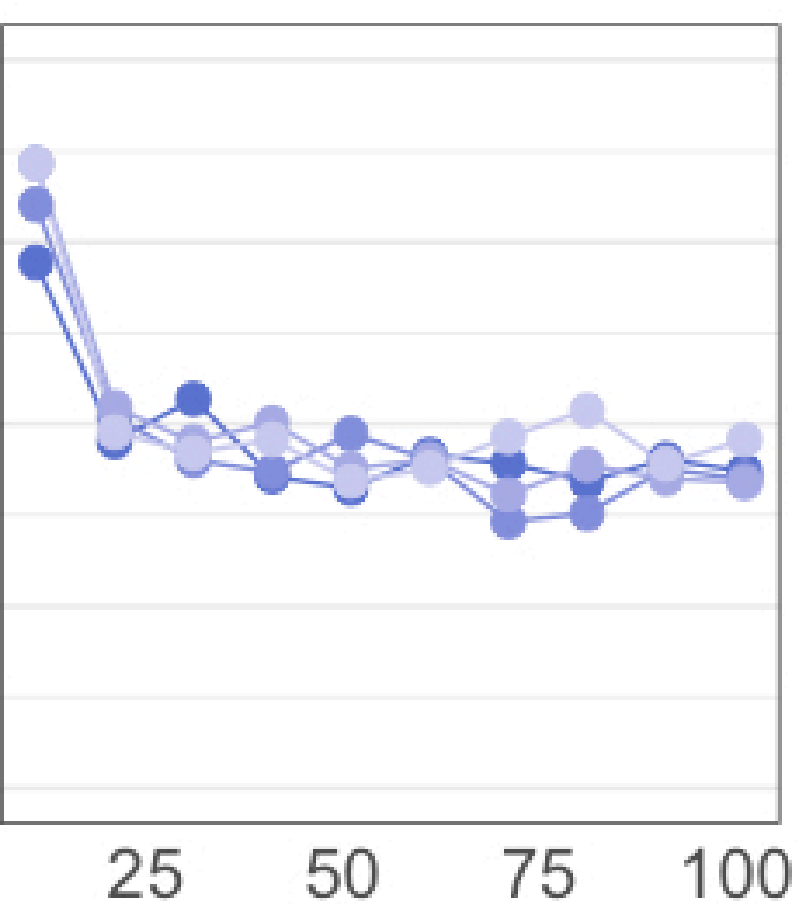

0.25

0.00 Seasons $r=0.001$

$$
r=0.04
$$

$$
r=0.1
$$

Patches with Dispersal

- $20 \%$ $40 \%$ $60 \%$ $80 \%$ 